\title{
Effect of ethnicity on vinorelbine pharmacokinetics: a population pharmacokinetics analysis
}

\author{
Aurélie Pétain ${ }^{1} \cdot$ Dafang Zhong $^{2} \cdot$ Xiaoyan Chen $^{2} \cdot{\text { Zhang } \mathrm{Li}^{3} \cdot \text { Shao Zhimin }}^{4} \cdot$ Jiang Zefei $^{5} \cdot$ Grégoire Zorza $^{1}$. \\ Pierre Ferré ${ }^{1}$ (1)
}

Received: 1 October 2018 / Accepted: 13 May 2019 / Published online: 27 May 2019

(c) The Author(s) 2019

\begin{abstract}
Background Pharmacokinetics of vinorelbine is mainly known from studies conducted in European patients. Interethnic differences in drug disposition may, however, induce interethnic variation in drug exposure. This paper aimed to evaluate the effect of ethnicity on the bioavailability and clearance of oral and intravenous vinorelbine.

Methods Oral and intravenous vinorelbine pharmacokinetics data in Asian patients were pooled from two-phase II studies of patients with non-small-cell lung cancer or advanced breast cancer in China. Blood vinorelbine and its active metabolite, 4'-O-deacetylvinorelbine, were quantified using liquid chromatography-tandem mass spectrometry. Bayesian pharmacokinetic parameters were calculated and vinorelbine monotherapy results (intravenous $25 \mathrm{mg} / \mathrm{m}^{2}$; oral $60 \mathrm{mg} / \mathrm{m}^{2}$ ) of the Asian data set were compared to a reference European data set (intravenous $30 \mathrm{mg} / \mathrm{m}^{2}$; oral $80 \mathrm{mg} / \mathrm{m}^{2}$ ). Subsequently, a population pharmacokinetics analysis was conducted in a combined cohort (Asian data set + historical vinorelbine pharmacokinetics database) to investigate for a potential effect of ethnicity.

Results Pharmacokinetics data from the Asian data set (oral: $n=47$; intravenous: $n=34$ ) was compared to the European reference data set (oral: $n=48$; intravenous: $n=48$ ). Mean apparent clearance of oral vinorelbine and mean absolute clearance of intravenous vinorelbine was comparable between the Asian and reference European data set. A population pharmacokinetic analysis (oral: $n=222$; intravenous: $n=111$ ) demonstrated no influence of ethnicity on oral and intravenous vinorelbine bioavailability and clearance.

Conclusion Vinorelbine pharmacokinetics were found to be comparable between Asian and European patients. No relevant influence of ethnicity on vinorelbine bioavailability and clearance for oral and intravenous routes of administration was observed.
\end{abstract}

Keywords Vinorelbine $\cdot$ Non-small-cell lung cancer $\cdot$ Breast cancer $\cdot$ China $\cdot$ Pharmacokinetics

Pierre Ferré

pierre.ferre@pierre-fabre.com

1 Institut de Recherche Pierre Fabre, 3 Avenue Hubert Curien, 31035 Toulouse, France

2 Shanghai Institute of Materia Medica, Chinese Academy of Science, Shanghai, People's Republic of China

3 Sun Yat-Sen University Cancer Center, Guangzhou, People's Republic of China

4 Fudan University Cancer Hospital, Shanghai, People's Republic of China

5307 Hospital of PLA, Beijing, People's Republic of China

\section{Introduction}

Vinorelbine is a vinca alkaloid that is widely used for the treatment of advanced or metastatic non-small-cell lung cancer (NSCLC) and advanced breast cancer (ABC). Vinorelbine has been marketed in France since 1989 and China since 1993 as an aqueous solution for intravenous administration (Navelbine ${ }^{\circledR}, 10 \mathrm{mg} / \mathrm{ml}$ ). A new oral formulation of vinorelbine has been developed as a line extension of the intravenous formulation for the same indications. Oral vinorelbine was developed as soft gelatin capsules and became available for clinical use in Europe since 2001, and more recently in China in 2014 [1].

During its clinical development, the pharmacokinetics of intravenous vinorelbine have been investigated and 
documented in a series of phase I $[2,3]$ and phase II studies [4]. The pharmacokinetics of intravenous vinorelbine is characterized by a large volume of distribution (between $70-75.61 \mathrm{l} / \mathrm{kg}$ ) [5, 6], reversible binding to blood platelets $(>70 \%)[5,7]$, and low protein binding (13\%) [8]. Pharmacokinetic characteristics of oral vinorelbine were obtained from traditional pharmacokinetic analysis within each clinical study, and from population pharmacokinetic analysis conducted on pooled data sets from clinical studies $[9,10]$. Oral vinorelbine is rapidly absorbed with an absolute bioavailability of approximately $40 \%$ [11]. Drug exposure is not influenced by food intake [12], and interindividual variability is the same as that for the intravenous formulation $[10,13]$. Reproducible drug exposures were observed over successive courses, and a dose-proportional increase in exposures was established [10, 14]. The pharmacokinetics of oral vinorelbine has been investigated in special sub-populations such as the elderly [15] and liver-impaired patients [16]. No dose adjustments of vinorelbine are required for elderly patients or patients with mild-to-moderate liver impairment $[15,16]$.

The equivalence between oral and intravenous doses required to achieve comparable blood exposure was established at 60 and $80 \mathrm{mg} / \mathrm{m}^{2}$ oral vinorelbine for 25 and $30 \mathrm{mg} / \mathrm{m}^{2}$ intravenous vinorelbine, respectively [11]. In a randomized phase II study, both formulations showed comparable activity and a qualitatively similar safety profile [4].

The metabolism pathway of vinorelbine primarily involves liver CYP3A4 enzymes to form inactive metabolites, except for, $4^{\prime}-O$-deacetylvinorelbine, the only active metabolite likely formed by carboxyl-esterases [17]. For both oral and intravenous administrations, bile is the major route of elimination for vinorelbine and its metabolites. Less than $10 \%$ of vinorelbine is eliminated via the urine, which mainly involves the parent compound [18].

Clinical studies of oral and intravenous vinorelbine have been mostly conducted in European patients. However, two-phase II studies have been completed in China to document oral and intravenous vinorelbine pharmacokinetics in Asian patients. One study was conducted in patients with ABC, and the other in patients with NSCLC. In both studies, patients were randomized to receive either intravenous or oral vinorelbine and the pharmacokinetic profile of vinorelbine was assessed. The objective of the present paper was to compare the pharmacokinetics of vinorelbine in Asian patients from pooled data of the twophase II studies in China with those of European patients reported in Bourgeois et al. [11]. A population pharmacokinetics analysis was then conducted to further evaluate the effect of ethnicity on the bioavailability and clearance of oral and intravenous vinorelbine.

\section{Methods}

\section{Description of clinical studies}

The present study is based on pooled pharmacokinetic data obtained from two prospective, multicenter, openlabel, randomized phase II trials conducted in $\mathrm{ABC}$ and NSCLC patients in China. Both studies were conducted in accordance with the Declaration of Helsinki and the International Conference on Harmonization Good Clinical Practice guidelines, local laws, and applicable regulatory requirements. The study protocols and their related documents (including the patient information and informed consent form) were approved by the local Ethics Committees and Competent Authority. All patients provided written informed consent for participation in the studies.

For the NSCLC study, key inclusion criteria were as follows: aged between 18 and 75 years (inclusive); men or non-pregnant, non-lactating women; cytologically or histologically confirmed diagnosis of NSCLC; stage IIIB, IV, or inoperable relapsed disease of any stage; and not previously treated with chemotherapy or immunotherapy. For the ABC study, key inclusion criteria were as follows: aged between 18 and 70 years (inclusive); non-pregnant, non-lactating women; and histologically confirmed adenocarcinoma of the breast. For both studies, eligible patients should have adequate bone marrow, hepatic and renal functions, and those over 65 years should not have more than three relevant co-morbidities that affect cardiac, pulmonary, liver, or renal functions.

Common key exclusion criteria for both studies were as follows: concomitant treatment with any other anticancer drugs; presence of malabsorption syndrome or disease significantly affecting gastro-intestinal function or major resection of the stomach or proximal small bowel that could affect absorption of oral vinorelbine; known hypersensitivity to drugs with similar chemical structures of study drugs; concomitant treatment with corticosteroids except chronic treatment lasting more than 1 month, given at low doses $(\leq 20 \mathrm{mg} /$ day of methylprednisolone or equivalent).

\section{Treatments}

Patients in both studies were randomized to receive either $60 \mathrm{mg} / \mathrm{m}^{2}$ oral or $25 \mathrm{mg} / \mathrm{m}^{2}$ intravenous vinorelbine during the first treatment cycle on days 1 and on days 8 . For oral administration, vinorelbine supplied as $20 \mathrm{mg}$ and $30 \mathrm{mg}$ capsules (Navelbine ${ }^{\circledR}$ Oral) were administered after meals in the presence of a healthcare professional. Patients were instructed not to chew or suck the capsules and to 
ingest the capsules rapidly in whole. In case of vomiting after oral administration, the study drug was not replaced. For intravenous administration, vinorelbine supplied as $10 \mathrm{mg} / \mathrm{ml}$ vials (Navelbine ${ }^{\circledR}$ i.v.) was reconstituted in normal saline aqueous solution and infused intravenously over 6-10 min after thorough dilution and under close supervision. In both studies, patients received vinorelbine alone on day 8 or in combination with capecitabine (ABC) or cisplatin (NSCLC), on day 1. Only pharmacokinetic data generated with vinorelbine given alone were used for ethnicity analysis in the current study.

\section{Pharmacokinetic assessment}

Blood samples were collected over $24 \mathrm{~h}$ during the first and second administrations of vinorelbine. Timepoints for blood sampling were as follows, according to the administered form. Oral vinorelbine: $0 \mathrm{~h}$ (pre-dose), $1.5 \mathrm{~h}, 3 \mathrm{~h}, 6 \mathrm{~h}, 11 \mathrm{~h}$, and $24 \mathrm{~h}$. Intravenous vinorelbine: $0 \mathrm{~h}$ (pre-dose), $3 \mathrm{~h}, 6 \mathrm{~h}$, $11 \mathrm{~h}$, and $24 \mathrm{~h}$. These sampling times included D-optimal sampling times previously determined [9]. At each sampling timepoint, $3 \mathrm{ml}$ of blood was collected in lithium heparin tubes. In patients receiving intravenous vinorelbine, blood samples were collected from the arm contralateral to the drug infusion arm. Blood samples were immediately frozen at $-20{ }^{\circ} \mathrm{C}$ and then stored at $-80{ }^{\circ} \mathrm{C}$ until analysis. Blood concentrations of vinorelbine and its metabolite, $4^{\prime}$ - $O$-deacetylvinorelbine, were quantified by a validated liquid chromatography-tandem mass spectrometry method [19] at SIMM Bioanalytical Laboratory (Shanghai, China). The lower limit of quantification was $0.25 \mathrm{ng} / \mathrm{ml}$ for both compounds.

\section{Pharmacokinetic data analysis}

\section{Calculation of individual pharmacokinetic parameters}

Empirical Bayes estimates of vinorelbine pharmacokinetic parameters were calculated using a previously developed population pharmacokinetic IV model [9] and an oral model [10] for, respectively, oral and IV data. Briefly, a linear three-compartment model with a zero-order infusion rate for intravenous vinorelbine and first-order absorption rate for oral vinorelbine was used to describe blood vinorelbine concentrations over time. Elimination from the central compartment was modelled with a first-order rate constant. Individual concentration vs. time data were fitted using the post hoc option in NONMEM ${ }^{\circledR}$ version 6.2 (ICON Development Solutions, Ellicott City, MD, USA). The overall goodness of fit between observed and predicted blood vinorelbine concentrations was evaluated through graphical appraisal of individual weighted residuals over time. Individual values of apparent total clearance $\left(\mathrm{Cl}_{\text {tot }} / F\right)$ for oral vinorelbine and absolute total clearance $\left(\mathrm{Cl}_{\text {tot }}\right)$ for intravenous vinorelbine were estimated and summarized by vinorelbine administration route (i.e., oral or intravenous) using descriptive statistics.

As the DVRL metabolite exhibited only very low concentration levels $(<10 \mathrm{ng} / \mathrm{ml})$, no model has been developed for DVRL. Graphically, no major difference between Asian and European population was evidenced (data not shown).

\section{Comparison between Asian data set and reference European data set}

Empirical Bayes estimates of vinorelbine pharmacokinetic parameters from the Asian data set were compared to those from a phase I pharmacokinetic study performed in Europe (reference European data set) [11]. The reference European data set was chosen, as it represented a robust and reliable evaluation of both oral and intravenous vinorelbine pharmacokinetics under strictly controlled and standardized conditions of administration similar to those in the studies of Asian patients. Furthermore, pharmacokinetic assessment from the reference European study was performed using the same bioanalytical method as that used in the present study. Details of the methodology used in the reference European data set have been previously published [11]. Pharmacokinetic parameters investigated included absolute total clearance $\left(\mathrm{Cl}_{\text {tot }}\right)$ for intravenous vinorelbine, apparent total clearance $\left(\mathrm{Cl}_{\text {tot }} / F\right)$ for oral vinorelbine, and inter-individual variability $(\mathrm{CV} \%)$ in bioavailability and $\mathrm{Cl}_{\text {tot }}$. Comparison of the pharmacokinetic parameters between the Asian and reference European data set was performed on log-transformed values using one-way analysis of variance. $p$ values $<0.05$ were considered statistically significant.

\section{Population pharmacokinetic modelling}

To further evaluate the potential effect of ethnicity on the pharmacokinetics of vinorelbine, the Asian data set (oral: $n=47$; intravenous: $n=34$ ) was combined with two large historical vinorelbine pharmacokinetic databases (oral/iv model: $n=175$ with $n=50$ iv data and 125 oral; iv model: $n=77$ ) built from several phase I and II studies performed in Europe and analyzed using the respective population pharmacokinetics models previously developed for each route of administration $[9,10]$. Concentration vs. time data were fitted using the non-linear mixed effect approach with the NONMEM $^{\circledR}$ program using the same estimation method than in the historical model (i.e., FO for oral model and FOCE for iv model). The models were first refined through estimation of a new set of parameters, including the significant covariate effects previously identified as relevant (e.g., body surface area, creatinine clearance as calculated by the Cockcroft and Gault formula, grade of transaminase 
elevation, and platelet count). Thereafter, ethnicity was tested as a new covariate and integrated into the final population pharmacokinetic model. The potential effect of ethnicity was tested on the typical values of vinorelbine clearance using the intravenous model, and on both clearance and bioavailability using the oral model.

Ethnic covariate was modelled as a power function of the typical pharmacokinetic parameter. For example, the ethnic covariate model for clearance parameter was

$\mathrm{Cl}_{i j}=\overline{\mathrm{Cl}} \cdot \theta^{\mathrm{cov}} \cdot e^{\eta i \mathrm{Cl}} \cdot e^{\eta \mathrm{Cl}}$,

where $\mathrm{Cl}_{i j}$ is the clearance $(\mathrm{Cl})$ of individual $i$ and occasion $j, \mathrm{Cl}$ is the typical value of $\mathrm{Cl}$ in the population, $\eta_{i \mathrm{Cl}}$ is the random inter-patient variability, $\eta_{j \mathrm{Cl}}$ is the random interoccasion variability, $\theta$ is the shift parameter describing the systematic dependence of the pharmacokinetic parameter on individual covariate value, and $\mathrm{Cov}$ is the individual covariate value [i.e., ethnicity coded either as 0 (European) or 1 (Asian)].

The relevance of a possible effect of the ethnicity was evaluated by several criteria:

1. Objective function value (OFV): a reduction in OFV of more than 3.84 from the reference model (excluding the ethnic covariate) was required to conclude a statistical significance at a nominal $\alpha$ risk of 5\% (log-likelihood ratio test).

2. Magnitude of the interaction effect: an effect of more than or equal to $20 \%$ was considered to be clinically relevant.

3. Precision of point estimate: the interaction effect and its 95\% confidence interval, as computed from the normal asymptotic theory using NONMEM $^{\circledR}$, were considered to be relevant if the $95 \%$ confidence interval did not include the null effect.

Correctness of each model (i.e., oral and intravenous models) was assessed by standard goodness of fit plots.

\section{Results}

\section{Patient characteristics}

Vinorelbine pharmacokinetic data from the $\mathrm{ABC}$ and NSCLC studies in Asian patients were pooled (oral: $n=47$; intravenous: $n=34$ ) and compared to the European reference data set (oral: $n=48$; intravenous: $n=48$ ). Comparison of patient characteristics between the Asian and European data sets showed lower body surface area and slightly higher creatinine clearance (Cockcroft and Gault formula) in Asian patients (Table 1). Other patient characteristics were similar between the two data sets.

\section{Comparison of vinorelbine pharmacokinetics between Asian and European patients}

Vinorelbine clearance was comparable between Asian and European patients regardless of the route of administration (Fig. 1). Although there was a slight trend towards a higher oral apparent clearance normalized to body surface area in Asian patients (median [range] 89.4 [25-250] 1/h/ $\mathrm{m}^{2}$ ) compared with European patients (median [range] 76.7 [26-235] $1 / \mathrm{h} / \mathrm{m}^{2}$ ), this trend did not reach statistical significance ( $p=0.07$; Fig. 1a). Intravenous vinorelbine clearance in Asian patients (median [range] 23.75 [9.87-40.42] 1/h/ $/ \mathrm{m}^{2}$ ) and European patients (median [range] 23.40 [13.55-43.74] $\left.1 / \mathrm{h} / \mathrm{m}^{2}\right)$ was found to be comparable ( $p=0.57$; Fig. $\left.1 \mathrm{~b}\right)$.

\section{Population pharmacokinetic analysis}

\section{Effect of ethnicity on vinorelbine pharmacokinetics}

Pharmacokinetics data from Asian patients were pooled with the historical European reference database for population pharmacokinetics analysis (oral: $n=222$; intravenous: $n=111$ ).

Population pharmacokinetic analysis was conducted in two steps. First, a "control" model was developed without
Table 1 Patient characteristics in Asian and European data sets

\begin{tabular}{|c|c|c|c|}
\hline & \multicolumn{2}{|l|}{ Asian } & \multirow{2}{*}{$\begin{array}{l}\text { European } \\
\text { Oral/intravenous }^{\mathrm{a}}(n=48)\end{array}$} \\
\hline & Oral $(n=47)$ & Intravenous $(n=34)$ & \\
\hline Gender (male/female) & $12 / 35$ & $9 / 25$ & $11 / 37$ \\
\hline Age (years) & $51[35-71]$ & $53.5[31-66]$ & $57.5[25-71]$ \\
\hline Body surface area $\left(\mathrm{m}^{2}\right)$ & $1.57[1.35-1.87]$ & $1.60[1.30-1.82]$ & $1.71[1.35-2.11]$ \\
\hline Creatinine clearance (ml/min) & $97.9[63.2-252]$ & $96.1[52.3-179]$ & 79.6 [35.9-127] \\
\hline
\end{tabular}

Data are presented as number or median [range]

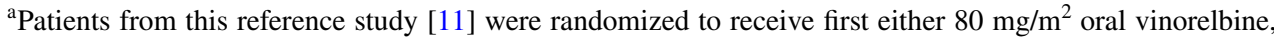
or $30 \mathrm{mg} / \mathrm{m}^{2}$ intravenous vinorelbine in a crossover study design with a 2 -week washout period. Hence, all 48 patients from the European data set received both oral and intravenous vinorelbine 
ethnicity as a covariate and using the same parameterization as historically (i.e., same fixed and random parameters and same residual error model). Descriptive statistics of the covariates are presented in Table 2. Next, ethnicity was introduced as a new covariate to the control model, resulting in a "test" model. Results of the population pharmacokinetic models are presented in Table 3. Goodness of fit of the final models including the ethnicity covariate indicates the model adequacy (Figs. 2, 3). Pred and variance-corrected VPC plots show that observations are included within the range of concentrations simulated with the models (Figs. 4, 5). (a)

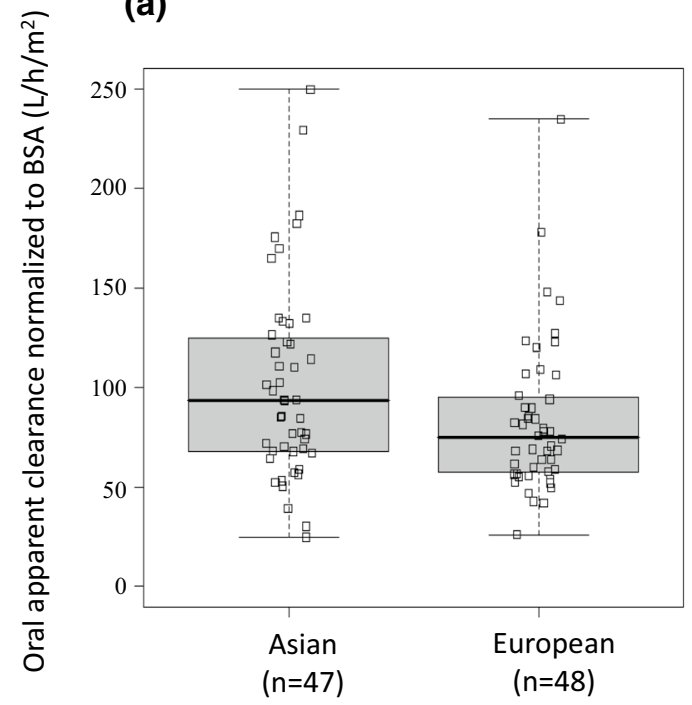

(b)

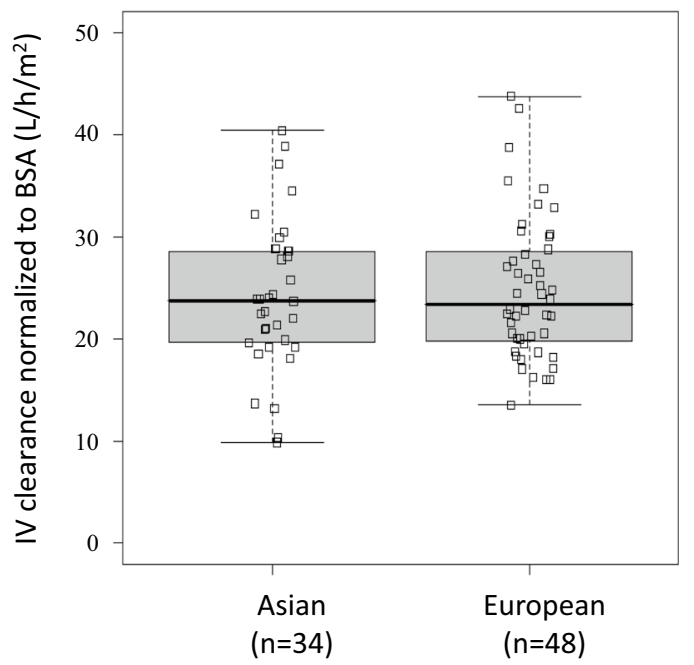

BSA = body surface area; IV = intravenous

Fig. 1 Comparison of $\mathbf{a}$ apparent total clearance $\left(\mathrm{Cl}_{\text {tot }} / F\right)$ of oral vinorelbine and $\mathbf{b}$ absolute total clearance $\left(\mathrm{Cl}_{\text {tot }}\right)$ of intravenous vinorelbine between Asian and European patients. $B S A$ body surface area, $I V$ intravenous

Table 2 Descriptive statistics of the covariates used in the population pharmacokinetic models

\begin{tabular}{llllll}
\hline Database covariate & \multicolumn{2}{l}{ Oral model } & & \multicolumn{2}{l}{ IV model } \\
\cline { 2 - 3 } \cline { 5 - 6 } & Asian & Historical & & Asian & Historical \\
\hline BSA & $1.57[1.35-1.87]$ & $1.72[1.32-2.33]$ & & $1.59[1.3-1.82]$ & $1.7[1.35-2.33]$ \\
Creatine clearance $<70 \mathrm{ml} / \mathrm{min}$ & $10.6 \%$ & $41 \%$ & & \\
Platelets & $250[103-659]$ & $267[90-540]$ & & \\
Transaminases grade $\geq 1$ & $10.6 \%$ & $18 \%$ & & \\
\hline
\end{tabular}

Table 3 Estimates from the population pharmacokinetic models for oral and intravenous vinorelbine

\begin{tabular}{|c|c|c|c|c|}
\hline & \multicolumn{2}{|c|}{ Oral vinorelbine $(n=222)$} & \multicolumn{2}{|c|}{ Intravenous vinorelbine $(n=111)$} \\
\hline & Control model & $\begin{array}{l}\text { Test model (with ethnicity covari- } \\
\text { ate on absolute bioavailability } \\
{[F] \text { ) }}\end{array}$ & Control model & $\begin{array}{l}\text { Test model (with } \\
\text { ethnicity covariate on } \\
\mathrm{Cl}_{\text {tot }} \text { ) }\end{array}$ \\
\hline Objective function value (OFV) & $17,819.5$ & $17,818.2$ & 5217.4 & 5216.7 \\
\hline Change in $\mathrm{OFV}^{\mathrm{a}}$ & - & -1.3 & - & -0.7 \\
\hline Inter-individual variability in $\mathrm{Cl}_{\text {tot }}(\mathrm{CV} \%)$ & 33.9 & 33.7 & 28.1 & 28.0 \\
\hline Inter-individual variability in Bioavailability (CV\%) & 20.5 & 20.6 & - & - \\
\hline Estimated magnitude of ethnic effect (\%) [95\% CI] & - & $5.3[-19.0$ to 29.6$]$ & - & $-5.1[-17.5$ to 7$]$ \\
\hline
\end{tabular}

${ }^{\mathrm{a}} \mathrm{A}$ decrease from the control model in OFV higher than 3.8 and 7.8 was needed to conclude to any statistically significant difference at $5 \%$ and $0.5 \%$ risk levels, respectively 

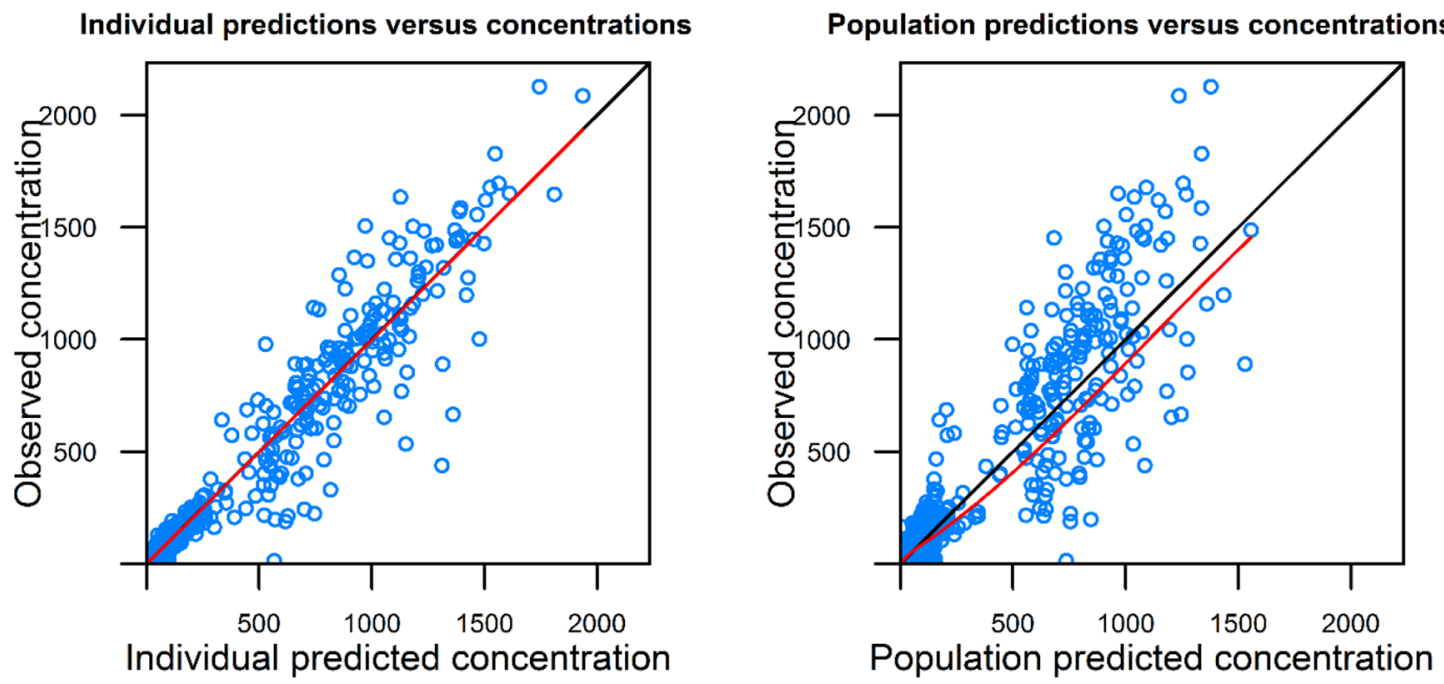

Conditional weighted residuals versus population predictions Conditional weighted residuals versus time
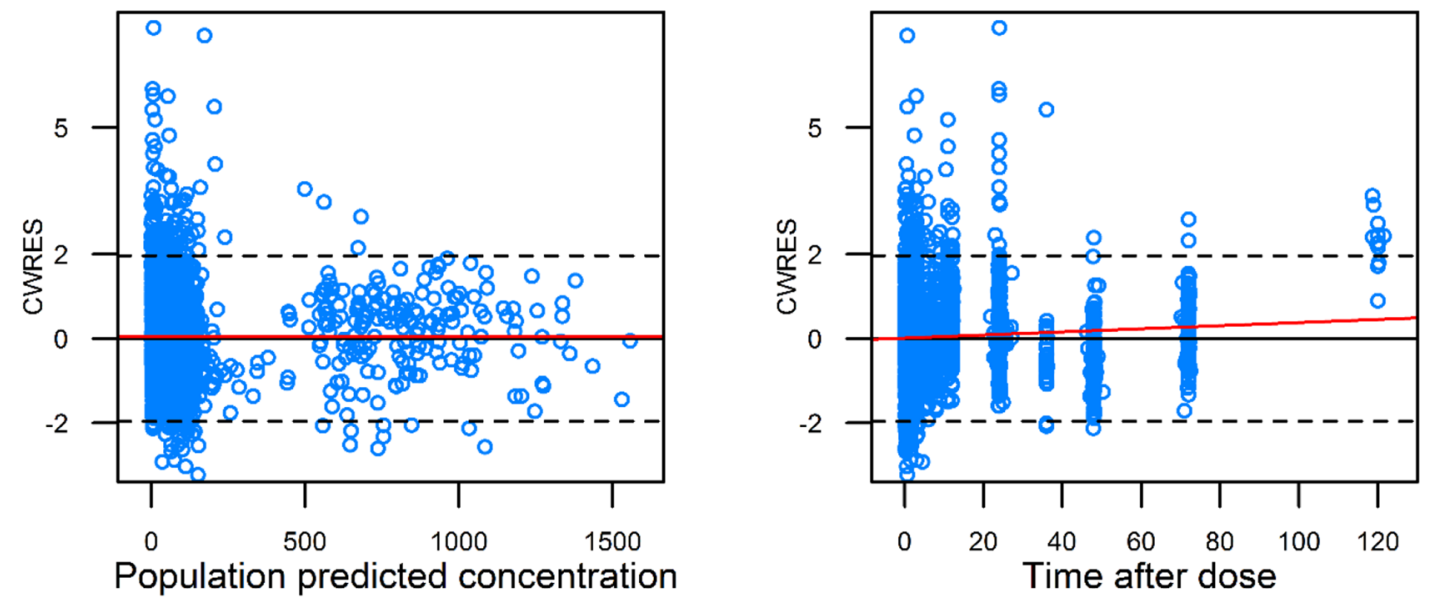

Fig. 2 Basic goodness of fit of oral model with ethnicity covariate. Concentrations are expressed as ng/ml; time is expressed as hours

For oral vinorelbine, inclusion of ethnicity as a covariate did not significantly improve the fit of the model (change in $\mathrm{OFV}=-1.3$; Table 2). Inter-individual variability in total clearance and bioavailability changed minimally from $33.90 \%$ (control model) to $33.70 \%$ (test model) and from 20.50 to $20.60 \%$, respectively. The estimated magnitude of the effect of ethnicity was not significantly different from zero $(5.3 \%$; $95 \%$ CI from bootstrap results: -20 to $306 \%$ ).

Likewise, for intravenous vinorelbine, inclusion of ethnicity as a covariate also did not significantly improve the fit of the model (change in $\mathrm{OFV}=-0.7$; Table 2). Inter-individual variability in total clearance changed minimally from $28.1 \%$ (control model) to $28.0 \%$ (test model). The estimated magnitude of the effect of ethnicity was not significantly different from zero $(-5.1 \%$; $95 \%$ CI from bootstrap results: -19.1 to $9.1 \%)$.

\section{Discussion}

The bioavailability and elimination of drugs are known to be influenced by ethnicity, especially for drugs which undergo hepatic metabolism [20]. Given that hepatic elimination is the main route of vinorelbine excretion [21], the potential ethnic differences in the pharmacokinetics of vinorelbine must be investigated. The present study compared pharmacokinetics data obtained from Asian patients to those from European patients to evaluate the potential influence of ethnicity on vinorelbine exposure. Vinorelbine clearance was not significantly different between Asian and European patients. Moreover, the population pharmacokinetic analyses give robust results and showed no relevant influence of ethnicity on vinorelbine clearance and bioavailability for both the oral and intravenous routes of administration. Overall, the pharmacokinetics of vinorelbine were found to 
Individual predictions versus concentrations

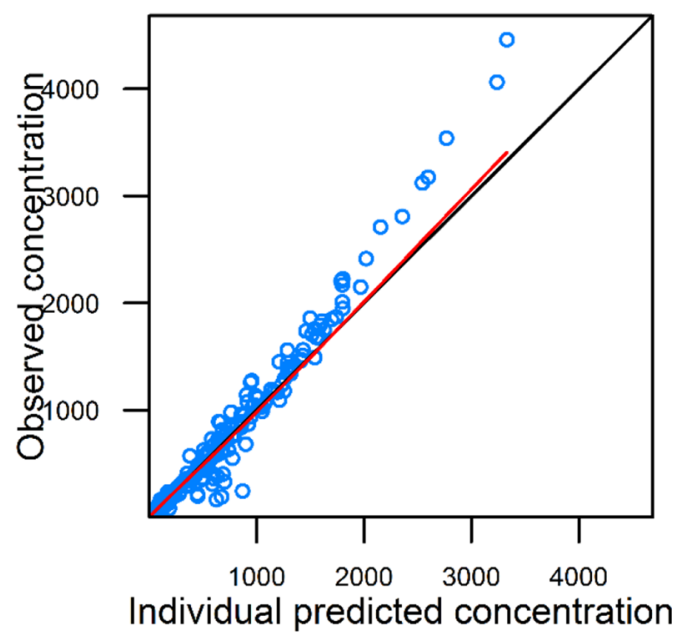

Population predictions versus concentrations

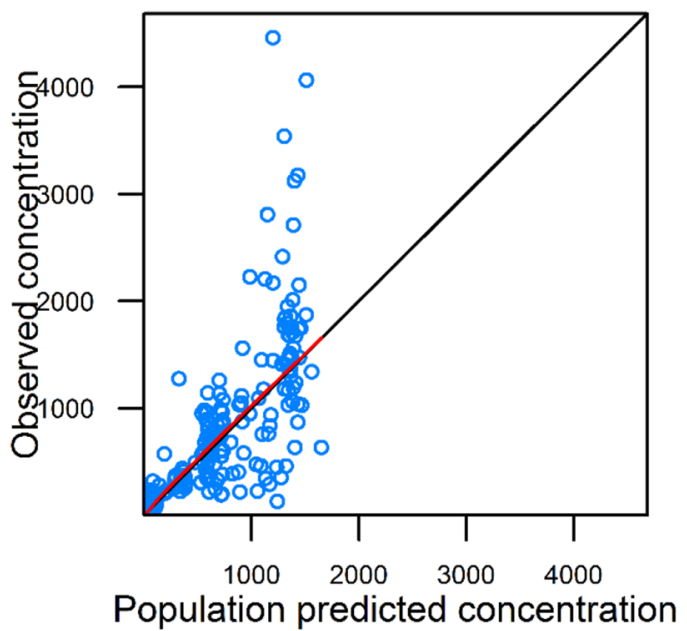

Conditional weighted residuals versus population predictions Conditional weighted residuals versus time
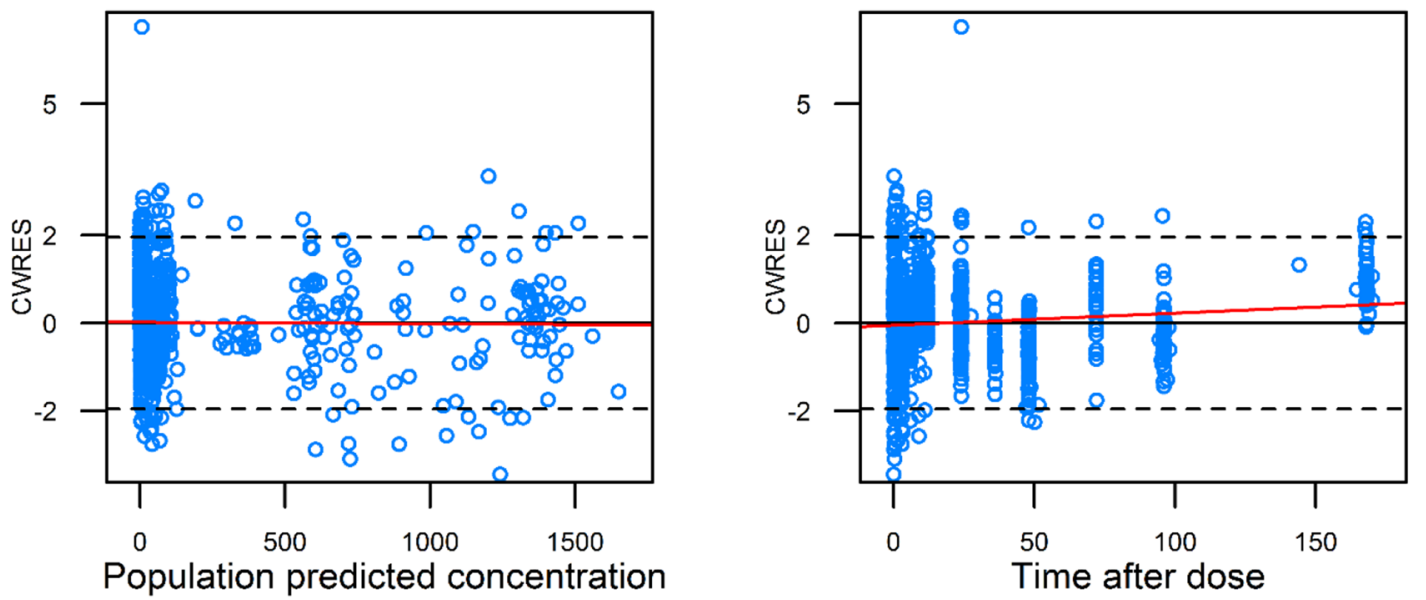

Fig. 3 Basic goodness of fit of IV model with ethnicity covariate. Concentrations are expressed as ng/ml; time is expressed as hours

be comparable between Asian and European patients, and data for the Asian patients were pooled from two-phase II studies conducted in China. Vinorelbine doses administered, blood sampling timepoints, duration of study, and bioanalytical methods used were similar in both studies. Although there were some slight differences in patient gender proportions between studies due to the cancer types studied, they were not expected to influence the pharmacokinetics of vinorelbine. Data for the European patients were obtained from a phase I pharmacokinetic study performed in Europe [11]. This reference European data set was selected for comparison, as it represented a robust and reliable evaluation of both IV and oral vinorelbine pharmacokinetics under strictly controlled and standardized conditions of administration. The intensive sampling schedule (12 timepoints) over a $168 \mathrm{~h}$ time period ensured a full description of the PK profile. The European trial also shared similar conditions of administration for both oral and IV vinorelbine with that of the Asian studies: oral vinorelbine was taken with food and with oral $5-\mathrm{HT}_{3}$ antagonist for anti-emetic treatment and IV vinorelbine was infused over a short 10 min period. Moreover, vinorelbine blood concentrations from the Asian studies were measured with the same bioanalytical method (LC/MS-MS method) as that used in the reference European trial; cross validation of the method was performed to ensure consistency in the results (internal data).

Patient characteristics of the Asian and European data sets were mostly comparable, with some expected exceptionsmedian body surface area of the Asian patients was significantly less than that of the European patients. To account for these differences, vinorelbine clearance values were adjusted for body surface area. Notably, the adjusted values did not differ significantly between the Asian and European data sets. It may be important to note that vinorelbine dosing 


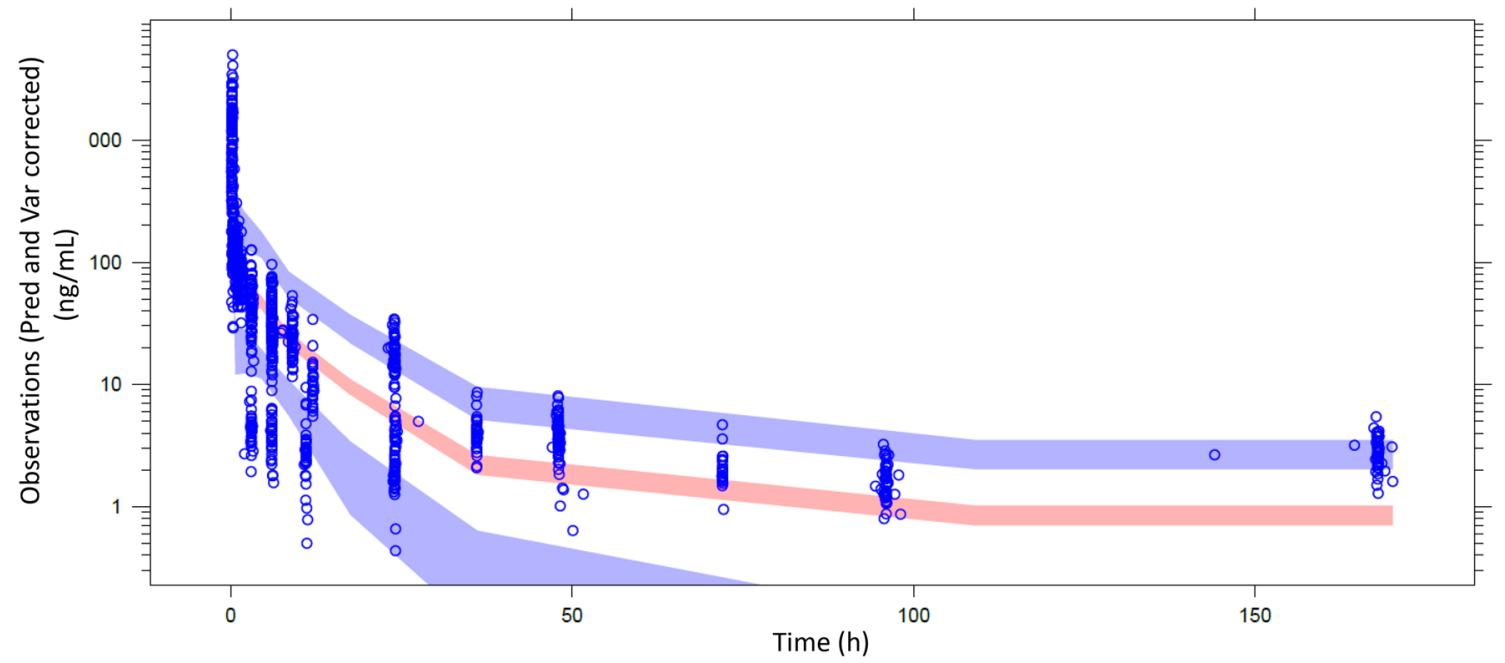

Fig. 4 Visual predictive check (IV model). Dot points represent observed values. Areas are, respectively, confidence interval of P5, median and P95 simulated values

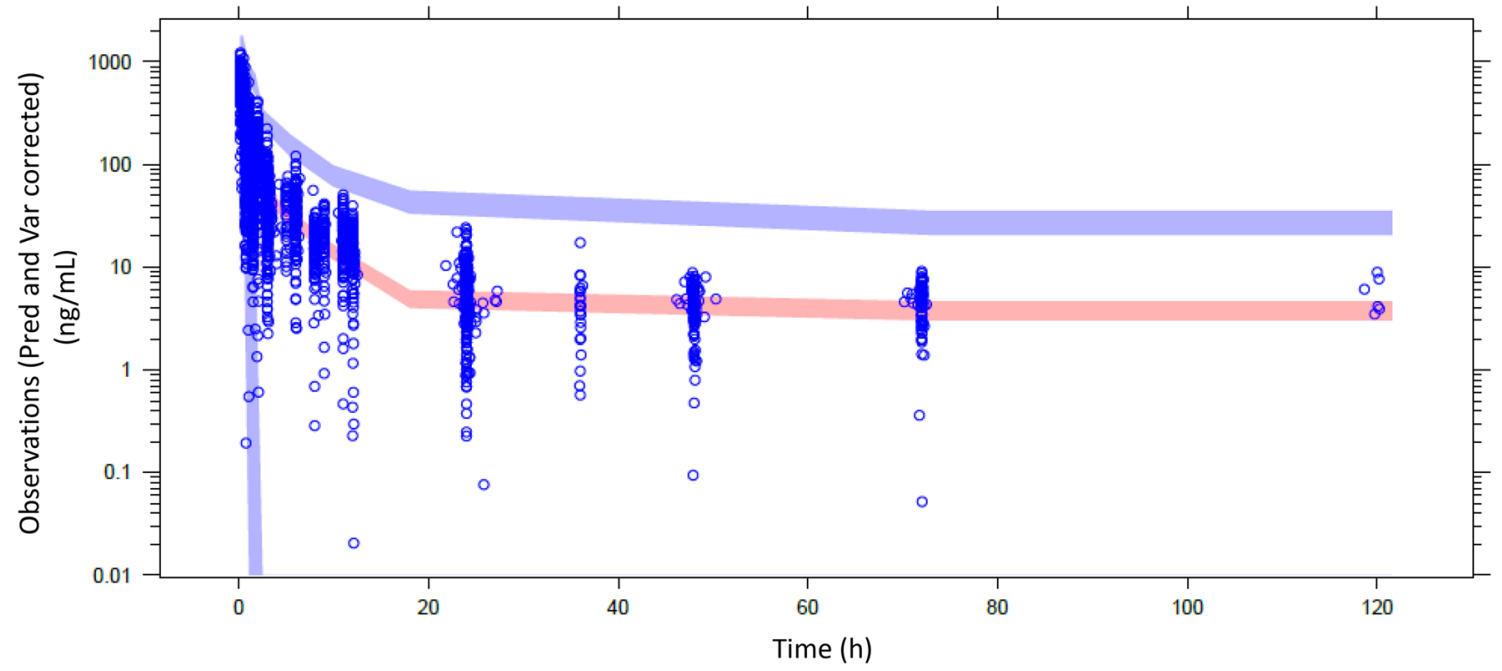

Fig. 5 Visual predictive check (oral model). Dot points represent observed values. Areas are, respectively, confidence interval of median and P95 simulated values. Confidence interval of P5 does not appear because of negative values

regimens differed between the Asian and European studies; oral and intravenous vinorelbine doses administered in the European trial were higher than those used in the Asian studies (oral: 80 vs. $60 \mathrm{mg} / \mathrm{m}^{2}$; IV: 30 vs. $25 \mathrm{mg} / \mathrm{m}^{2}$ ) [11]. However, since the pharmacokinetics of vinorelbine is linear [8], vinorelbine clearance is not expected to be dependent on the dose administered.

Ethnic differences in drug bioavailability and metabolism are known to cause variability in responses to anticancer drugs [22]. One example is that of vincristine, a vinca alkaloid, which is metabolized more efficiently by CYP3A5 than by CYP3A4 enzymes. Ethnic differences in expression of
CYP3A5 between ethnic groups (70\% prevalence in African Americans vs. $20 \%$ in Caucasians) resulted in significant variability in vincristine-associated neurotoxicity $(4.8 \%$ vs. $34.8 \%$, respectively) [23]. Caucasians also experienced higher grades of toxicity and were more likely to have their vincristine doses reduced or omitted than African Americans [23]. Based on the comparison between Asian and European data sets, the present analysis suggests comparable vinorelbine clearance between Asian and European patients. Further evaluation by population pharmacokinetic analysis in large historical vinorelbine pharmacokinetic databases showed that ethnicity was not a relevant covariate for 
absolute bioavailability, as its inclusion into the oral model did not show any significant improvement of fit. There was also no decrease in inter-individual variability when the ethnic covariate was integrated into the model. This is in line with the metabolism knowledge of vinorelbine, mainly involving esterase and CYP3A4 enzymes [24], which are not described as highly functionally polymorphic in the European and Asian populations.

Assuming the absence of co-morbidities, similarities in pharmacokinetic behaviors between Asian and European patients suggest that similar dosage regimen of vinorelbine may be used in both populations. Furthermore, duplication of effort and cost may be avoided as data for either of these populations could be bridged to the other.

In conclusion, the results obtained from the comparison between Asian and European data sets and population pharmacokinetic analysis demonstrated that the pharmacokinetics of oral and IV vinorelbine was not different between Asian and European patients. Knowledge of the pharmacokinetic behavior of vinorelbine in these populations could help to improve individualization of treatment regimens in terms of therapeutic outcomes and safety.

Acknowledgements This study was funded by Pierre Fabre Médicament, France. Medical writing and editorial support was funded by Pierre Fabre Médicament, France, and provided by Wei Yi Kwok and Bao Hui Lee from Tech Observer Asia Pacific Pte. Ltd.

\section{Compliance with ethical standards}

Conflict of interest AP, GZ, and PF are employees of Pierre Fabre Research Institute. All other authors have no conflict of interest to disclose.

Ethical standards All procedures performed in studies involving human participants were conducted in accordance with the Declaration of Helsinki and the International Conference on Harmonization Good Clinical Practice guidelines, local laws, and applicable regulatory requirements.

Informed consent Informed consent was obtained from all patients who participated in the studies.

Open Access This article is distributed under the terms of the Creative Commons Attribution 4.0 International License (http://creativeco mmons.org/licenses/by/4.0/), which permits unrestricted use, distribution, and reproduction in any medium, provided you give appropriate credit to the original author(s) and the source, provide a link to the Creative Commons license, and indicate if changes were made.

\section{References}

1. Pierre Fabre Press Release (2014, Sept 24) Pierre Fabre Médicament obtains MA in China for NAVELBINE ${ }^{\circledR}$ Oral (Vinorelbine) in the treatment of advanced lung and breast cancer. https ://www.pierre-fabre.com/en/news/pierre-fabre-medicament -obtains-ma-in-china-for-navelbineroral-vinorelbine

2. Johansen M, Kuttesch J, Bleyer WA et al (2006) Phase I evaluation of oral and intravenous vinorelbine in pediatric cancer patients: a report from the Children's Oncology Group. Clin Cancer Res 12(2):516-522

3. Schilling T, Fiebig HH, Kerpel-Fronius S et al (1996) Clinical phase I and pharmacokinetic trial of vinorelbine administered as single intravenous bolus every 21 days in cancer patients. Invest New Drugs 14(4):371-378

4. Jassem J, Ramlau R, Karnicka-Mlodkowska H et al (2001) A multicenter randomized phase II study of oral vs. intravenous vinorelbine in advanced non-small-cell lung cancer patients. Ann Oncol 12(10):1375-1381

5. Leveque D, Jehl F (1996) Clinical pharmacokinetics of vinorelbine. Clin Pharmacokinet 31(3):184-197

6. Marquet P, Lachatre G, Debord J et al (1992) Pharmacokinetics of vinorelbine in man. Eur J Clin Pharmacol 42(5):545-547

7. Urien S, Bree F, Breillout F et al (1993) Vinorelbine high-affinity binding to human platelets and lymphocytes: distribution in human blood. Cancer Chemother Pharmacol 32(3):231-234

8. Gebbia V, Puozzo C (2005) Oral versus intravenous vinorelbine: clinical safety profile. Expert Opin Drug Saf 4(5):915-928

9. Nguyen L, Tranchand B, Puozzo C, Variol P (2002) Population pharmacokinetics model and limited sampling strategy for intravenous vinorelbine derived from phase I clinical trials. $\mathrm{Br}$ J Clin Pharmacol 53(5):459-468

10. Variol P, Nguyen L, Tranchand B, Puozzo C (2002) A simultaneous oral/intravenous population pharmacokinetic model for vinorelbine. Eur J Clin Pharmacol 58(7):467-476

11. Bourgeois H, Vermorken J, Dark G et al (2007) Evaluation of oral versus intravenous dose of vinorelbine to achieve equivalent blood exposures in patients with solid tumours. Cancer Chemother Pharmacol 60(3):407-413

12. Bugat R, Variol P, Roche $\mathrm{H}$ et al (2002) The effects of food on the pharmacokinetic profile of oral vinorelbine. Cancer Chemother Pharmacol 50(4):285-290

13. Marty M, Fumoleau P, Adenis A et al (2001) Oral vinorelbine pharmacokinetics and absolute bioavailability study in patients with solid tumors. Ann Oncol 12(11):1643-1649

14. Bonneterre J, Chevalier B, Focan C, Mauriac L, Piccart M (2001) Phase I and pharmacokinetic study of weekly oral therapy with vinorelbine in patients with advanced breast cancer (ABC). Ann Oncol 12(12):1683-1691

15. Puozzo C, Gridelli C (2004) Non-small-cell lung cancer in elderly patients: influence of age on vinorelbine oral pharmacokinetics. Clin Lung Cancer 5(4):237-242

16. Kitzen JJ, Puozzo C, de Jonge MJ, Brandely M, Verweij J (2010) Mild to moderate liver dysfunction does not require dose reduction of oral or intravenous vinorelbine: results of a pharmacokinetic study. Eur J Cancer 46(2):266-269

17. Beulz-Riche D, Grude P, Puozzo C et al (2005) Characterization of human cytochrome P450 isoenzymes involved in the metabolism of vinorelbine. Fundam Clin Pharmacol 19(5):545-553

18. Krikorian A, Rahmani R, Bromet M, Bore P, Cano JP (1989) Pharmacokinetics and metabolism of navelbine. Semin Oncol 16(2 Suppl 4):21-25

19. Van Heugen JC, De Graeve J, Zorza G, Puozzo C (2001) New sensitive liquid chromatography method coupled with tandem mass spectrometric detection for the clinical analysis of vinorelbine and its metabolites in blood, plasma, urine and faeces. $\mathbf{J}$ Chromatogr A 926(1):11-20

20. Johnson JA (1997) Influence of race or ethnicity on pharmacokinetics of drugs. J Pharm Sci 86(12):1328-1333 
21. Wargin WA, Lucas VS (1994) The clinical pharmacokinetics of vinorelbine (navelbine). Semin Oncol 21(5 Suppl 10):21-27

22. O'Donnell PH, Dolan ME (2009) Cancer pharmacoethnicity: ethnic differences in susceptibility to the effects of chemotherapy. Clin Cancer Res 15(15):4806-4814

23. Renbarger JL, McCammack KC, Rouse CE, Hall SD (2008) Effect of race on vincristine-associated neurotoxicity in pediatric acute lymphoblastic leukemia patients. Pediatr Blood Cancer 50(4):769-771

24. de Graeve J, van Heugen JC, Zorza G, Fahy J, Puozzo C (2008) Metabolism pathway of vinorelbine (navelbine) in human: characterisation of the metabolites by HPLC-MS/MS. J Pharm Biomed Anal 47(1):47-58

Publisher's Note Springer Nature remains neutral with regard to jurisdictional claims in published maps and institutional affiliations. 